\title{
Kernos
}

Revue internationale et pluridisciplinaire de religion grecque antique

15 | 2002

Varia

\section{Le syncrétisme d'Asclépios avec le Cavalier Thrace}

\section{Ivanka Dontcheva}

URL : http://journals.openedition.org/kernos/1391

DOI : 10.4000/kernos.1391

ISSN : 2034-7871

\section{Éditeur}

Centre international d'étude de la religion grecque antique

\section{Édition imprimée}

Date de publication : 1 janvier 2002

ISSN : 0776-3824

\section{Référence électronique}

Ivanka Dontcheva, "Le syncrétisme d'Asclépios avec le Cavalier Thrace », Kernos [En ligne], 15 | 2002, mis en ligne le 21 avril 2011, consulté le 20 avril 2019. URL : http://journals.openedition.org/ kernos/1391; DOI : 10.4000/kernos.1391 


\section{Le syncroétisme d'Asclépios avec le Cavalier Thrace}

Asclépios est une des divinités auxquelles on avait consacré le plus grand nombre de sanctuaires dans le monde gréco-romain. Pausanias en mentionne soixante-trois, mais d'après certains auteurs, la propagation du culte d'Asclépios fut si large, qu'au cours du II $^{\mathrm{e}}$ siècle ap. J.-C., il y avait 320 sanctuaires en activité ${ }^{1}$. À la période romaine, cette ample propagation du culte ne dépasse pas les régions thraces. Une des divinités les plus vénérées en Thrace, après le Cavalier Thrace, a été Asclépios. Les témoignages de son culte, découverts dans les régions thraces, sont variés et innombrables, d'ordre archéologique, épigraphique et numismatique ${ }^{2}$. Un des témoignages de la propagation particulièrement large de ce culte est le fait que, des vingtcinq villes qui ont frappé monnaie en Thrace, seules huit n'ont pas utilisé l'effigie d'Asclépios.

La diffusion large et facile du culte d'Asclépios parmi la population autochtone était due aux fonctions qu'il partageait avec la divinité principale thrace, connue sous le nom de Cavalier Thrace. Les sanctuaires thraces les plus connus, liés au culte d'Asclépios, sont ceux de Glava Panega ${ }^{3}$, Batkoun ${ }^{4}$, Pernik $^{5}$, Slivnitza ${ }^{6}$, Pautalia $^{7}$, mais des témoignages de ce culte ont été

1 J. BeAujEau, La religion romaine à l'apogée de l'Empire. La politique religieuse des Amtonins, Paris, 1955, p. 301.

2 Iv. Dontcheva, Le culte d'Asclépios en Thrace [dissertation en bulgare], Sofia, 2000.

3 V. Doвruskı, "Le sanctuaire thrace d'Asclépios près de'Glava Panega" [en bulgare], Bulletin arcbéologique du Musée national 1 (1907), p. 3-86; Z. GočEvA, "Das Heiligtum des Thrakischen Reiters bei Glava Panega und die Probleme der Romanisierung von Moesia Inferior", Balcanica Posnaniensia 7 (Poznan, 1995), p. 141-156.

4 D. Tzontchev, Le sanctuaire thrace près du village de Batkoun len bulgare], Sofia, 1941.

5 V. Lubenova, "Le sanctuaire près du Pernik" [en bulgare], Monuments thraces 2 (Sofia, 1980), p. 15-48.

6 D. Boteva, "Le sanctuaire thrace d'Asclépios Limenos près de Slivnitza. Monuments votifs et cultuels du sanctuaire" [en bulgare], Recherches étudiantes, VII-1 (Sofia, 1985), p. 23-93; M. Tatcheva, "Le sanctuaire thrace d'Ascépios Limenos près de la ville Slivnitza et ses archives épigraphiques" [en bulgare], Thracia Antiqua 10 (In memoriam Georgi Mibailov), Sofia, 1999, p. 152-170.

7 J. Ivanov, "Le Hisarlak de Kustendil et ses antiquités" [en bulgare], Bulletin de la Société arcbéologique bulgare 7 (Sofia, 1919-1920), p. 66-124. 
découverts dans beaucoup d'autres endroits - à Odessos ${ }^{8}$, Serdica ${ }^{9}$, Germania $^{10}$, Nicopolis ad Istrum ${ }^{11}$, Philippopolis ${ }^{12}$, Karassura ${ }^{13}$, etc.

Il faut souligner que, dans les centres urbains, Asclépios apparaît sous son aspect gréco-romain, ce qui est attesté par son iconographie traditionnelle hellénistique ainsi que par les épithètes fonctionnelles employées pour vénérer le dieu-guérisseur. Par contre, dans les sanctuaires situés hors des villes, il est vénéré en relation avec le Cavalier Thrace, surtout dans son aspect de dieu suprême et de défenseur contre tous les maux. Ce syncrétisme est très bien exprimé dans les particularités iconographiques des reliefs votifs découverts dans ces sanctuaires, ainsi que dans les épithètes locales qui accompagnaient le nom d'Asclépios dans les inscriptions votives. Ce sont les particularités iconographiques de ce type de monuments qui seront le centre d'intérêt de notre étude.

L'iconographie d'Asclépios se distingue nettement de celle de la plupart des grands dieux du panthéon antique à cause de sa personnalité et des conditions de son accession au premier plan - au moment où s'amorçait le déclin de la plastique religieuse ${ }^{14}$. Très vite, son iconographie se limite à reproduire les quelques types statuaires créés au Iv ${ }^{\mathrm{e}}$ siècle av. J.-C. Dans l'art plastique, Asclépios apparaît d'abord sur les reliefs attiques immédiatement postérieurs à son installation à Athènes à la fin du v $\mathrm{v}^{\mathrm{e}}$ siècle av. J.-C., à un moment où son iconographie est encore instable. Mais très vite, les reliefs reproduisent les types statuaires qui font autorité. Dès lors, l'iconographie d'Asclépios est presque exclusivement statuaire. L'inventivité se trouve limitée à quelques variations autour des statues de culte les plus célèbres, surtout celles d'Épidaure (représentant Asclépios trônant) et d'Athènes (Asclépios debout). Dans ces conditions, les représentations d'Asclépios ne diffèrent que par l'attitude, voire seulement la pondération ou la disposition du drapé de son himation. On remarque cette extraordinaire uniformité jusqu'à la périphérie du monde romain, où l'on ne décèle presque aucune

8 Iv. Dontcheva, "Le culte d'Asclépios à Odessos" [en bulgare], Thracia 12 (Studia in bonorem Christo M. Danov), Serdicae, 1998, p. 181-193.

9 B. Gerov, "Recherches sur les terres thraces occidentales à l'époque romaine" [en bulgare], Annuaire de l'Université de Sofia, Faculté des Lettres 62, 2 (Sofia, 1968), p. 183185 (en bulgare); G. Mihallov, IGBulg. IV, nº 1934-1936.

10 Zv. Dremsizova, "Une trouvaille de Sapareva bania" [en bulgare], Bulletin de l'Institut arcbéologique bulgare 18 (Sofia, 1952), p. 353-358.

11 Iv. Dontcheva, "Le culte d'Asclépois à Nicopolis ad Istrum" [en bulgare], Thracia Antiqua 10 (In memoriam Georgi Mibailov), Sofia, 1999, p. 170-177.

12 B. Diakovitch, "La frise des divinités guérisseuses de la Thrace. Le culte d'Esculape" [en bulgare], Annuaire du Musée national archéologique Plovdiv, 1921, Plovdiv, 1923, p. 127-178.

13 Iv. Dontcheva, "Les témoignages sur le culte d'Asclépios à Carassura" [en bulgare], Seminarium Tbracicum 3 (Sofia, 1998), p. 147-157.

14 B. HoltzmanN, art. Asclepios, LIMC II, 1 (1984), p. 863-897. 
particularité iconographique ${ }^{15}$. Ce n'est qu'en terre thrace que l'on rencontre une iconographie différente, représentant Asclépios à cheval, qui n'existe nulle part dans le monde antique. Cette iconographie purement thrace est le résultat du syncrétisme d'Asclépios avec la divinité la plus vénérée des Thraces, le Cavalier Thrace.

Dans les régions thraces, on distingue deux types iconographiques d'Asclépios sur les reliefs votifs ${ }^{16}$. Le premier suit fidèlement l'iconographie gréco-romaine du dieu-guérisseur et on peut la voir surtout dans les centres urbains (Pautalia, Serdica, Nicopolis ad Istrum, Philipoppolis, Odessos), tandis que le deuxième type reflète un aspect local du culte, qui emprunte l'iconographie et, avec elle, les épithètes locales du Cavalier Thrace. On peut la voir surtout dans les sanctuaires ruraux (Glava Panega, Batkoun, Pernik, Slivnitza). Les représentations du premier type qui suivent l'iconographie traditionnelle d'Asclépios peuvent être classées en plusieurs groupes: 1) Asclépios seul; 2) Asclépios avec Hygie; 3) Asclépios avec Télesphore; 4) Asclépios avec Hygie et Télesphore; 5) Asclépios avec d'autres divinités ${ }^{17}$. Notre étude portera sur les représentations d'Asclépios du deuxième type iconographique, qui est fortement influencé par une iconographie purement thrace et qui illustre le syncrétisme d'Asclépios avec le Cavalier Thrace.

La lente infiltration et l'établissement du culte d'Asclépios dans les sanctuaires thraces et, par la suite, sa fusion avec le Cavalier Thrace sont bien attestées par l'évolution de l'iconographie des reliefs votifs. Un des premiers reliefs qui témoigne de la vénération simultanée d'Asclépios et du Cavalier Thrace avant leur syncrétisme complet a été découvert près du village Izvorovo, dans la région de Tchirpan ${ }^{18}$. Il s'agit du seul monument de ce genre. Sur le relief, entouré d'un cadre dont la partie supérieure est légèrement cintrée et ressemble à un fronton triangulaire, sont représentés le Cavalier Thrace, Asclépios et Hygie. Au centre du relief, entre le Cavalier Thrace d'un côté et Asclépios et Hygie de l'autre, est représenté un autel sur lequel brûle un feu. À gauche du relief, on voit le Cavalier Thrace se diriger vers l'autel brûlant. Il est vêtu d'un habit court aux longues manches et d'un pantalon très étroit. Sur son dos, on voit une chlamyde drapée. Son visage, tourné aux trois quarts, est encadré d'une courte barbe bouclée et d'une moustache. Dans la main droite, collée au cou du cheval, il tient une phiale. À droite de l'autel sont représentés Asclépios et Hygie selon leur iconographie traditionnelle. Sur le relief, il n'y a pas d'inscription dédicatoire, mais

15 Ibid., p. 866.

16 Z. Gočeva, art. Asclepios (in Thracia), LIMC II, 1 (1984), p. 897-901; Iv. DontchevA, "Caractéristique iconographique des représentations d'Asclépios sur les reliefs votifs thraces" [en bulgare], in Recbercbes bistoriques et arcbéologiques in memoriam du prof. dr Stantcbo Vaklinov, Véliko Tirnovo, 1994, p. 323-335.

17 Dontcheva, ibid., p. 326-330.

18 G. Kazarow, Die Denkmäler des thrakischen Reittergottes in Bulgarien, Budapest, 1938, p. 428, fig. 238. 
de la scène représentée on peut déduire que le dédicant était d'origine thrace. C'est la représentation du Cavalier Thrace qui appuie cette hypothèse. Dans ce cas-là, le dédicant a fait une dédicace à la fois au Cavalier Thrace, à Asclépios et à Hygie. L'autel allumé, représenté entre le Cavalier Thrace et les divinités de la santé, ainsi que la phiale dans la main du cavalier symbolisent l'acte de l'offrande et l'obtention de la bienveillance divine. La taille égale des trois divinités démontre qu'elles étaient considérées comme égales en importance. La représentation simultanée d'Asclépios et du Cavalier Thrace prouve que les deux divinités ayant des fonctions similaires étaient vénérées d'une façon indépendante et que le syncrétisme entre elles n'était pas encore réalisé. Mais la représentation inhabituelle du Cavalier Thrace avec la barbe est une preuve que le processus était déja amorcé.

Les reliefs les plus intéressants, découverts dans le sanctuaire thrace près de Batkoun, illustrent l'étape initiale du syncrétisme du culte local avec celui d'Asclépios. Sur ces reliefs Asclépios est représenté à cheval ${ }^{19}$. Un des ces reliefs présente un intérêt particulier, parce qu'Asclépios est représenté selon son iconographie traditionnelle gréco-romaine ${ }^{20}$. Il est vêtu de son himation long, rejeté par-dessus l'épaule gauche et laissant nus son torse, son épaule et sa main droite. Ce qui est inhabituel dans ce cas, c'est qu'Asclépios est à cheval galopant vers droite. Dans sa main droite, le dieu tient son attribut habituel, le bâton avec le serpent enroulé. Ce qui surprend, c'est que le serpent lui aussi est représenté avec la barbe, un attribut typique d'Asclépios. Devant le cheval, on voit un autel allumé. Dans l'art gréco-romain, Asclépios n'est jamais représenté à cheval, ce qui vaut également pour les autres dieux du panthéon antique. Sur ce relief, on voit le début de la fusion des figures divines, proches dans leurs fonctions. On doit considérer cette étape comme un début, parce que l'on continue à représenter Asclépios selon son iconographie typique. Dans les étapes ultérieures, la représentation d'Asclépios emprunte entièrement l'iconographie du Cavalier Thrace et on le voit apparaître comme un jeune homme imberbe, vêtu d'un chiton court, parfois nu, et avec la chlamyde traditionnelle sur le dos. Mais bien avant d'emprunter l'iconographie du Cavalier Thrace, Asclépios avait emprunté ses épithètes locales, comme c'est le cas avec l'épithète $Z v \mu v \delta \rho \eta v o s$, liée au culte originel du sanctuaire près de Batkoun. Dans ce même sanctuaire, Asclépios figure sur deux autres reliefs, traités selon la même iconographie - il est à cheval, porte la barbe et l'himation ${ }^{21}$.

Un seul relief votif, découvert dans le sanctuaire du Batkoun, représente le Cavalier Thrace sous le nom d'Asclépios, accompagné d'une autre épithète

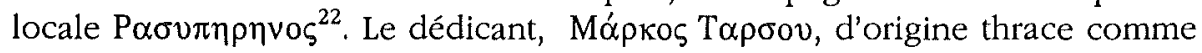

Tzontchev, o.c. (n. 4), p. 42-43, nº 1, 2, 3, 4, pl. XXXI, 124, 125, 126, 127.

Ibid., p. 42, $\mathrm{n}^{\circ} 1$, pl. XXXI, 124.

Ibid., p. 42-43, n² 2, 4, pl. XXXI, 125, 127.

DoBruski, l.c. (n. 3), p. 90, $\mathrm{n}^{\circ} 139$, fig. 69; IGBulg. III-1, $\mathrm{n}^{\circ} 1185$. 
l'atteste le nom de son père, était selon toute probabilité venu d'une autre

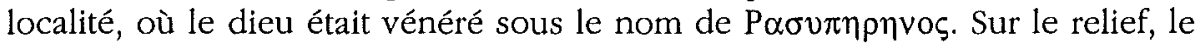
Cavalier Thrace est représenté avec la barbe et ses cheveux sont ceints de la corona tortilis, ce qui du point de vue iconographique le rapproche d'Asclépios et témoigne de l'étape suivante du syncrétisme entre les dieux. Ici, le cavalier n'est pas représenté vêtu d'un himation comme à l'étape initiale, mais porte un court chiton ceint et une chlamyde. Dans la main droite, au lieu du bâton avec le serpent enroulé, le cavalier tient une phiale appuyée contre le cou du cheval.

Asclépios est représenté comme un cavalier barbu sur plusieurs reliefs votifs dans le sanctuaire thrace de Glava Panega, consacré à Asclépios $\Sigma \alpha \lambda \delta \circ \beta v \sigma \tau \eta v \varsigma^{23}$. L'un d'eux présente un très grand intérêt car on y voit sous le cheval, entre ses sabots, un serpent rampant vers l'autel représenté devant le cheval ${ }^{24}$.

Les reliefs votifs mentionnés prouvent la transition progressive de la représentation d'Asclépios sous les traits du Cavalier Thrace, c'est-à-dire le syncrétisme complet entre le culte local thrace et celui d'Asclépios.

Un relief votif ${ }^{25}$ présente, selon nous, un intérêt particulier : le Cavalier Thrace tient dans la main droite, derrière la tête du cheval, une lyre, l'un des attributs d'Apollon. Au-dessus de la tête du cavalier apparaît un nimbe en forme de coquillage (Cardium). Sur le cadre supérieur du relief est gravée la dédicace à Asclépios. Ce monument reflète un syncrétisme composite. D'un côté apparaît le lien entre le Cavalier Thrace et Apollon à travers la lyre, et de l'autre, avec Asclépios, par l'inscription. Le nimbe en forme de coquillage est un attribut typique des nymphes et d'Aphrodite, et prouve de manière indéniable le rapport entre la divinité, les eaux et leur force vivifiante. La même représentation du Cavalier Thrace se trouve sur plusieurs reliefs votifs, consacrés à Asclépios $\mathrm{K} \varepsilon \imath \lambda \alpha \delta \varepsilon \eta v o \zeta$, découverts dans le sanctuaire de Pernik ${ }^{26}$, où le culte local était lié au culte d'Asclépios. D'autre part, le monument mentionné ${ }^{27}$ témoigne que, dans le sanctuaire, le culte d'Apollon était célébré, mais qu'il ne s'est pas imposé.

Sur les reliefs votifs qui représentent le Cavalier Thrace, mais portent des dédicaces à Asclépios, on remarque toujours la présence de l'attribut le plus typique d'Asclépios, le serpent. On le voit, soit enroulé autour du bâton qui remplace la lance dans la main droite du cavalier, soit rampant entre les sabots du cheval, soit enroulé autour d'un arbre qui se trouve devant le cavalier. Un intérêt particulier est présenté par certains reliefs votifs où le

27 Tzontchev, o.c. (n. 4), p. 53-54, n 40, pl. XLVII, 177; IGBulg. III-1, nº 1242.
} 
serpent rampe à côté du cheval. De tels reliefs ont été découverts dans les sanctuaires de Glava Panega et de Pernik et, dans ces cas, le serpent se dirige vers l'autel représenté devant le cheval. Le serpent en tant qu'attribut d'Asclépios et du Cavalier Thrace a une signification polysémique ${ }^{28}$. Au début, il était non seulement une incarnation d'Asclépios lui-même, mais il était aussi son premier auxiliaire et allié, en tant que personification des forces créatrices de la nature. Le lien du serpent avec le monde souterrain fait de lui un attribut typique des divinités chthoniennes, donc il symbolise à la fois la vie, la sagesse et la fertilité. En tant qu'attribut d'Asclépios, le serpent est non seulement une répercussion de son image thériomorphe initiale, mais il symbolise sa force guérisseuse, tout en se mettant au service du traitement des malades. Comme un bon génie, il protège la vie qu'il peut très facilement ravir et sa mue annuelle symbolise le retour à la vie du malade guéri.

Sur les reliefs votifs, qui représentent Asclépios sous les traits du Cavalier Thrace, apparaissent les trois types iconographiques de la classification de G. Kazarov ${ }^{29}$ : a) cavalier au repos ou au pas à droite; b) cavalier chasseur et c) cavalier revenant de la chasse. Les inscriptions dédicatoires nous permettent d'identifier la divinité figurée comme Asclépios. Il est représenté, soit la main droite levée, soit tenant la phiale appuyée au cou du cheval ${ }^{30}$. Parfois, dans sa main droite levée, il brandit une lance et pourchasse le gibier - le plus souvent un sanglier qui symbolise les forces du mal dans la nature ${ }^{31}$. Parfois, il figure rentrant de la chasse, le gibier tué à la main. Sur certains reliefs votifs, le gibier est attaqué par un ou deux chiens, parfois par un lion ${ }^{32}$.

Sur les reliefs votifs qui représentent Asclépios comme Cavalier Thrace, est figuré presque toujours un arbre avec un serpent enroulé autour de lui. Très souvent, près de l'arbre, est figuré un autel sur lequel le cheval pose le sabot. Dans certains cas, à part le cavalier, sont représentées d'autres figures humaines - une ou deux femmes devant le cheval ${ }^{33}$ ou un homme derrière lui. Sur d'autres reliefs est représentée une urne renversée, dont s'écoule de l'eau.

28 J. Schouten, The Road and Serpent of Asklepios (Symbol of Medicine), Amsterdam, 1967; J. Prieur, Les animaux sacrés dans l'antiquité, Rennes, 1988, p. 78-90; Iv. VENEdIKov, "Le Héros Thrace" [en bulgare], in Légendes thraces, Sofia, 1981, p. 30-31.

29 KAZAROW, o.c. (n. 18), p. 5-8.

30 Z. GočEva, "Les traits caractéristiques de l'iconographie du Cavalier Thrace", $B C H$, suppl. 14 (1986), p. 237-243.

31 Iv. Marazov, "Le sujet 'Chasse au sanglier'” [en bulgare], Arcbeologia (Sofia, 1975), 2 , p. $30-42$.

32 M. Oppermanne, "À propos des plaques votives du Cavalier Thrace chassant de l'époque romaine en Bulgarie" [en bulgare], Archeologia (Sofia, 1970), 2, p. 19-32.

33 T. Stojanov, "À propos de l'iconographie et de la sémantique des représentations féminines dans les monuments du Cavalier Thrace" [en bulgare], Vekove (Sofia, 1987), 1, p. $18-30$. 
Certains reliefs votifs consacrés à Asclépios $K \varepsilon \imath \lambda \alpha \delta \varepsilon \eta v o \zeta$ du sanctuaire de Pernik ${ }^{34}$ présentent un intérêt tout particulier. Les inscriptions dédicatoires et la figuration du cavalier, armé d'une lance, d'un bouclier et d'une épée sont un témoignage du rôle de la divinité comme protectrice de l'armée et des militaires. L'aspect guerrier du cavalier est souligné aussi sur plusieurs reliefs votifs, consacrés à Asclépios $\Lambda \imath \mu \eta v o s$ dans le sanctuaire de Slivnitza, où le cavalier est figuré avec une épée et coiffé d'un casque ${ }^{35}$.

Parmi les reliefs votifs, découverts dans les sanctuaires thraces les mieux étudiés - Glava Panega, Batkoun, Pernik, Slivnitza, - la plupart représentent le Cavalier Thrace, mais portent une inscription dédicatoire à Asclépios. C'est un témoignage clair que, dans ce sanctuaire thrace, on célébrait la figure locale, qui, à l'époque romaine, avait fusionné avec celle d'Asclépios. Toutes les inscriptions dédicatoires conservées sur les reliefs votifs représentant le Cavalier Thrace sont adressées à Asclépios, ce qui traduit la fusion complète entre les deux divinités.

La découverte d'une si grande quantité de monuments votifs consacrés à Asclépios montre la très large diffusion de son culte. La plastique statuaire, les reliefs votifs et les pièces de monnaies ${ }^{36}$ mis au jour dans les grands centres urbains suivent l'iconographie traditionnelle gréco-romaine du dieuguérisseur. Mais dans les sanctuaires ruraux d'Asclépios, on remarque la prédominance de l'iconographie typiquement thrace. Une autre preuve de la fusion des deux divinités, qui en principe avaient des fonctions similaires de dieux sauveurs et protecteurs, sont les épithètes locales accompagnant le nom d'Asclépios dans les inscriptions ${ }^{37}$. Toutes ces épithètes locales

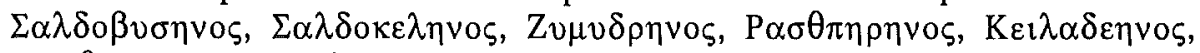

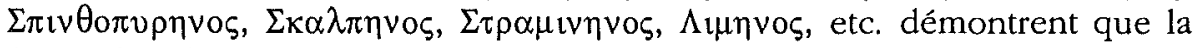
figure d'Asclépios avait fusionné avec le dieu thrace ancien.

Un autre fait est intéressant à souligner. La diffusion du culte d'Asclépios à la place de celui du Cavalier Thrace n'a pas entraîné la représentation d'Hygie et de Télésphore dans le cercle formé par l'entourage du Cavalier. Ces deux divinités lui restent étrangères. Un seul relief votif connu, découvert dans le sanctuaire de Glava Panega, représente le Cavalier Thrace avec une inscription dédicatoire à Asclépios, et représente Télesphore dans le fronton du cadre du relief ${ }^{38}$.

\footnotetext{
34 LuBenova, l.c. (n. 5), p. 30, nº $31,48$.

35

BOTEVA, l.c. (n. 6), p. 85.

36 R. Gouchtérakliev, "Représentations d'Asclépios, d'Hygie et de Télésphore sur les monnaies $\left(\mathrm{I}^{\mathrm{e}}-\mathrm{nII}{ }^{\mathrm{e}} \mathrm{s}\right.$ ). "[en bulgare], Bulletin du Musée bistorique de Lovetch 2 (Lovetch, 1996), p. 18-38.

37 Iv. Dontcheva, "Les épithètes d'Asclépios en Thrace et les aspects du culte qu'elles reflètent" [en bulgare], in Paleobalkanistique et paleobulgaristique. Deuxièmes lectures nationales d'automne "Prof. Ivan Galabov", Véliko Tirnovo, 2001, p. 551-564.

Doвruski, l.c. (n. 3), p. 56, fig. 28.
} 
Le problème du syncrétisme de ce dieu protecteur thrace, qui est connu sous le nom de Cavalier Thrace, est un problème fondamental pour la compréhension de la religion thrace. En plus d'Asclépios, d'autres divinités du panthéon gréco-romain sont aussi représentées à cheval - Apollon, Silvain, Zeus, Dionysos, Arès, mais toutes ces divinités occupent la place d'un seul et même dieu local qui, sous les épithètes locales les plus diverses, a emprunté la personnalité du Cavalier Thrace ${ }^{39}$. Il est évident que la représentation de ce dieu, tantôt sous les traits originaux du Cavalier Thrace, tantôt sous l'iconographie de ces divinités qui l'ont remplacé, a provoqué ce mélange d'attributs et même parfois le mélange des figures de ces dieux. C'est pourquoi l'iconographie présente certaines irrégularités et des exceptions à la règle générale et qui sont caractéristiques de la Thrace. Cependant rien n'y est dû au hasard. Derrière ces images se cache le syncrétisme thrace qu'il n'est pas toujours facile de reconnaître. Il faut souligner un fait étrange : Asclépios, Apollon, Silvain etc, ne remplacent pas des Asclépios, des Apollon et des Silvain thraces, mais occupent toujours la place d'une même divinité, le Cavalier Thrace. Cela n'eût pas été possible si les Thraces avaient eu de nombreuses divinités différentes, dont les fonctions coïncidaient avec celles des dieux grecs. Un culte individuel de toutes ces divinités du panthéon gréco-romain était une chose nouvelle et non encore complètement assimilée par les Thraces ${ }^{40}$. À l'appui de cette hypothèse viennent aussi les épithètes

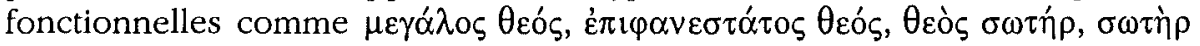

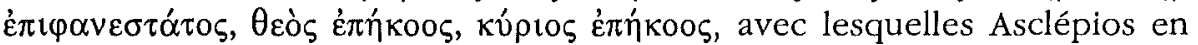
syncrétisme avec le Cavalier Thrace est vénéré dans les régions thraces, et qui soulignent son aspect de dieu suprême, protecteur et sauveur de toutes sortes de maux ${ }^{41}$. Un tel aspect du culte d'Asclépios en Thrace est en réalité à l'unisson avec les tendances générales de l'époque, à savoir l'universalisation et la panthéisation du culte d'Asclépios à travers le monde gréco-romain ${ }^{42}$.

Université "Sts. Cyrille et Méthode ",

Ivanka DonTCHEVA

Faculté d'Histoire, Chaire d'Archéologie

5003 Véliko TiRnovo

Bulgarie

39 I. Venedikov, "Le syncrétisme religieux en Thrace à l'époque romaine", in Acta antiqua Philippopolitana, Studia archaeologica, Serdicae, 1963, p. 153-166.

40 lbid., p. 166.

41 Dontcheva, l.c. (n. 37).

42 M. Le Glay, "Hadrien et l'Asclépieion de Pergame", BCH 100 (1976), p. 347-372. 